\title{
Altered Fate of Subventricular Zone Progenitor Cells and Reduced Neurogenesis following Neonatal Stroke
}

\author{
Ruggero Spadafora $^{a}$ Fernando F. Gonzalez $^{a, b}$ Nikita Derugin ${ }^{c}$ \\ Michael Wendland $^{d}$ Donna Ferriero ${ }^{a, b}$ Patrick McQuillen ${ }^{a, b}$ \\ Departments of a Neurology, ${ }^{b}$ Pediatrics, ' Neurosurgery, and ${ }^{\mathrm{d}}$ Radiology, University of California, \\ San Francisco, Calif., USA
}

\section{Key Words}

Ischemia $\cdot$ Neonate $\cdot$ Stem cells $\cdot$ Neurogenesis

\begin{abstract}
Objective: To investigate the effects of neonatal stroke on progenitor cells lining the lateral ventricles. Methods: Intraventricular injection of replication-incompetent green fluorescent protein (GFP)-expressing lentivirus was performed in postnatal day 1 (P1) rats to specifically label radial glia/ type $B$ neural stem cells and ependymal cells of the lateral ventricle. A subset of animals was exposed to transient middle cerebral artery occlusion (MCAO) at P7, with mild or moderate injury confirmed by diffusion-weighted MRI and histology. Newborn cells were identified by GFP expression, location and expression of cell type-specific markers in the striatum, cortex and olfactory bulb using confocal microscopy and systematic random sampling. Results: Three weeks lentiviral GFP transduction of cells in the lateral ventricle, abundant GFP-expressing neurons and glia were identified in the rostral migratory stream, olfactory bulb and striatum as expected from labeling the subventricular zone (SVZ) type B neural stem cell lineage. Two weeks following mild or severe focal stroke at P7, no GFP-expressing neurons were detected in striatum or cortex although some single-labeled
\end{abstract}

doublecortin+ immature neurons were detected in the penumbra. The densities of GFP+/ glial fibrillary acidic protein (GFAP)+ astrocytes and GFP+/O4+ oligodendrocytes were reduced in the striatum following MCAO $(4.8 \pm 1.02$ vs. 2.5 \pm 0.4 cells/high-power field, HPF; $p=0.005 ; 2.8 \pm 1$ vs. 0.5 \pm 0.2 cells/HPF, $p=0.008$ ). Furthermore, there was a reduction of GFP+ cells in the olfactory bulb following MCAO (58.8 \pm 14.9 vs. $19.6 \pm 5.4$ cells/HPF, $p=0.025)$. Finally, there was an increased percentage of GFP+/GFAP+ cells (70 vs. $50 \%$ ), with a decreased proportion of GFP+/O4+ cells (14 vs. $30 \%$ ) in injured animals. Conclusion: Neurogenesis originating from cells of the lateral ventricle, including SVZ type B cells, is significantly reduced following neonatal stroke. Furthermore, neonatal stroke disrupts gliogenesis in the striatum, decreasing overall numbers of new glia and shifting the population towards astrocytes.

Copyright $\odot 2010$ S. Karger AG, Basel

\section{Introduction}

Recent studies have documented significant postnatal neurogenesis in rodent models of ischemic brain injury [1-3], prompting great interest in its potential therapeutic capability. Newborn cells in these models are thought to

\section{KARGER}

Fax +4161306 1234 E-Mail karger@karger.ch www.karger.com (c) 2010 S. Karger AG, Basel

0378-5866/10/0322-0101\$26.00/0

Accessible online at:

www.karger.com/dne
Prof. Donna M. Ferriero, MD

University of California, San Francisco

521 Parnassus Avenue, Rm C215

San Francisco, CA 94143-0663 (USA)

Tel. +1 415502 5820, Fax +1 415502 5821, E-Mail donna.ferriero@ ucsf.edu 
originate from the subventricular zone (SVZ), a structure lining the cerebral ventricles. Following neonatal hypoxia-ischemia and adult stroke, the immature neurons emerge from the SVZ, migrate toward the site of injury and differentiate into region-appropriate neurons [1-4]. A number of methods have been used to quantify the SVZ response to both hypoxia-ischemia and ischemic stroke, including measurements of SVZ volumes [3, 5], expression of SVZ neural stem cell (NSC) markers such as nestin [3], and quantification of cell proliferation using thymidine analogs such as bromodeoxyuridine (BrdU) $[1,2]$. These techniques, however, are limited by problems related to cell fusion [6], DNA repair $[7,8]$ and a lack of specificity of the markers used to identify immature cells [9]. Surprisingly, little is known regarding the precise relationship of newborn cells to specific SVZ cell lineages.

The SVZ, along with the hippocampal dentate gyrus, is one of two well-characterized sources of newly generated neurons that persist throughout the life of the rodent [10]. Under normal conditions, the SVZ generates immature neurons (type A cells) that join the rostral migratory stream (RMS) and migrate tangentially towards the olfactory bulb, where they differentiate into mature interneurons [11]. In the first few weeks of postnatal life, the SVZ also generates astrocytes and oligodendrocytes that migrate radially towards overlying structures, including neocortex and intermediate zones, to complete a sequence of glial development that is initiated during embryonic life $[12,13]$. During embryonic and early postnatal life, radial glia are the stem cell population which differentiates soon after birth into adult NSCs that express glial fibrillary acidic protein (GFAP), also referred to as type B cells $[14,15]$. Radial glia are characterized by their apical and basal processes that project to the cortical and ventricular walls, the latter connection is maintained postnatally by type B cells [16]. The final groups of cells in this lineage are referred to as type $\mathrm{C}$ or transit-amplifying cells, proliferating cells that have a high mitotic rate and are directly generated by type B cells [17].

In this study, we sought to directly label all the cells of this lineage based upon this unique persistent contact maintained with the lateral ventricular wall by SVZ type B cells through a basal process [16]. This was accomplished by injecting the lateral ventricle immediately after birth with a lentiviral vector carrying a constitutively expressed gene for green fluorescent protein (GFP) [18]. Lentivirus infects both dividing and nondividing cells, and is therefore a highly efficient system to transduce all cells in contact with the ventricle, including SVZ type B NSCs [19]. Type B cells are slowly dividing cells and there- fore not highly susceptible to infection by retroviral vectors previously used $[3,19]$. Ependymal cells are a second population that will be labeled by intraventricular injection and may also represent a NSC population [20]. Our analysis focused on identification of the progeny of labeled cells in the olfactory bulb and striatum 3 weeks following viral injection. To determine the effect of neonatal stroke on this cell lineage, we exposed a second group of lentiviral-labeled animals to transient middle cerebral artery occlusion (tMCAO) on postnatal day 7 (P7), a model we developed [21] to mimic neonatal stroke in fullterm infants.

\section{Materials and Methods}

All protocols followed the guidelines of the Laboratory Animal Resource Center at the University of California, San Francisco, Calif., USA.

\section{GFP-Lentiviral Production}

Packaging plasmid pCMV-dR8.91 ( $\Delta 8.9)$ and expressing envelope plasmid VSV-G were transfected with the reporter plasmid FG12 [18], containing the gene encoding for GFP under the human Ubiquitin-C promoter, into 50\% confluent 293FT cells along with Effectene transfection reagent according to the manufacturer's instructions (Qiagen). After $48 \mathrm{~h}$, the viral supernatant was collected and centrifuged at 2,000 rpm for $10 \mathrm{~min}$ at $4^{\circ} \mathrm{C}$ to remove cell debris. The virus was concentrated 100 -fold by ultracentrifugation at $75,000 \mathrm{rpm}$ for $90 \mathrm{~min}$ at $4^{\circ} \mathrm{C}$, resuspended in PBS and stored at $-80^{\circ} \mathrm{C}$ until use. To measure titers, serially diluted lentivirus was used to transduce 293FT cells; $72 \mathrm{~h}$ later, cells expressing GFP were counted to calculate viral titer. Lentivirus with titers ranging from $1 \times 10^{6}$ to $1 \times 10^{7} \mathrm{CFU} / \mathrm{ml}$ was used in this study.

\section{Intraventricular Injections}

Newborn Long-Evans rat pups were obtained from Simonson Labs (Gilroy, Calif., USA). The dam and litter were given food and water ad libitum and housed in a temperature/light controlled animal care facility. Twelve postnatal-day-1 (P1, day of birth designated $\mathrm{P} 0$ ) rats were anesthetized by $3 \%$ isoflurane and placed into a stereotactic frame modified for neonatal rodents (Stoelting). The head was further stabilized by a customized head mold. The skull was exposed and the right lateral ventricle was targeted at the following coordinates from bregma: $1.0 \mathrm{~mm}$ anterior, 1.2 $\mathrm{mm}$ lateral, $2 \mathrm{~mm}$ depth. Injections were made with a beveled pulled glass micropipette (Wiretrol $5 \mu$ l, Drummonds Scientific Company, Broomal, Pa., USA) with a 50- to 70- $\mu$ m diameter tip positioned at an angle of $90^{\circ}$ to the surface of the skull to avoid passing through the striatum and the striatal SVZ. Two microliters of replication-incompetent lentivirus was injected at a rate of $0.5 \mu \mathrm{l} / \mathrm{min}$, and the tip was left in place for $2 \mathrm{~min}$ following the injection. Animals were then returned to the dam and monitored until they resumed nursing. 


\section{Newborn MCAO}

Ten P7 pups underwent MCAO and 4 sham surgery, as previously described [21]. Two pups died in the week following the procedure and 2 did not satisfy the injury criteria by diffusionweighted (DW)-MRI (see below), leaving 6 animals that were included in the MCAO group. Briefly, surgery was performed on spontaneously breathing pups anesthetized with $1.75 \%$ isoflurane in a mixture of $70 \% \mathrm{~N}_{2} \mathrm{O}$ and $30 \% \mathrm{O}_{2}$. The internal carotid artery (ICA) was dissected and a temporary ligature was applied at its origin using a 6-0 silk suture. A second silk suture was looped around the ICA, just above the pterygopalatine artery, and retracted laterally to prevent retrograde blood flow. A small arteriotomy was made in the proximal isolated ICA segment, and a coated 6-0 Dermalon filament was inserted and advanced 7.5$8.5 \mathrm{~mm}$, depending on the animal's weight, and secured with a temporary suture. Injury patterns were identified by DW-MRI during occlusion immediately prior to reperfusion. Four animals with the mild injury pattern, showing subcortical involvement, were reperfused after 90 min of occlusion by removing both sutures and the filament, after which the arteriotomy site was covered with Surgicel. An additional 2 animals demonstrated moderate/severe injury manifested by subcortical and cortical involvement. Histopathological findings confirmed mild injury selectively involving the striatum, as compared with moderate and moderate/severe injury characterized by cortical involvement and signs of infarction respectively, as previously described $[22,23]$.

\section{Magnetic Resonance Imaging}

MRI was performed using a 2-tesla magnet equipped with a Bruker Omega system and actively shielded gradients that provide $\pm 200 \mathrm{mTm}^{-1}$ gradient amplitude. The instrument settings for T2W and DW sequences were as previously described. Pups were anesthetized with $1.5-2 \%$ isofluorane $/ 100 \% \mathrm{O}_{2}$, placed supine on a plastic support, and water recirculating warming pads were wrapped around the pup below the neck to maintain body temperature. Pups were inserted into a $3.8-\mathrm{cm}$ diameter birdcage imaging coil. DW spin echo MRI was conducted $1.5 \mathrm{~h}$ after $\mathrm{MCAO}$ to confirm the presence of injury, as previously described. Eight consecutive 1-mm coronal sections were acquired, and the 6 that best covered the MCA territory beginning at the anterior edge of the forceps minor corpus callosum were used. Animals with DW hyperintensity in the basal ganglia ipsilateral to the occlusion but not in the neocortex were included in the study.

\section{BrdU Labeling}

Beginning on postoperative day 3, intraperitoneal injections of BrdU (50 mg/kg; Sigma, St. Louis, Mo., USA) were given twice daily for 3 days (P10-P12).

\section{Immunohistochemistry}

Animals were deeply anesthetized with sodium pentobarbital (100 mg/kg; Nembutal, Abbot Labs, Abbot Park, Ill., USA), and brains were harvested after transcardial perfusion with ice-cold $4 \%$ paraformaldehyde in $0.1 \mathrm{M}$ PBS ( $\mathrm{pH} 7.4$ ). Brains were carefully removed and postfixed overnight, equilibrated in $30 \%$ sucrose in $0.1 \mathrm{M} \mathrm{PBS}$ and left at $4^{\circ} \mathrm{C}$ for a maximum of $72 \mathrm{~h}$. Immunofluorescent staining was performed on $50-\mu \mathrm{m}$ free-floating coronal sections, which were collected throughout the brain using a sliding microtome. Sections were stored at $4^{\circ} \mathrm{C}$ in $0.1 \mathrm{M}$ phos- phate buffer with $0.1 \%$ sodium azide for a maximum of 2 weeks or stored in cryoprotectant at $-20^{\circ} \mathrm{C}$ until staining.

The following antibodies were used: anti-doublecortin (antiDCx; rabbit polyclonal, 1:200; Cell Signaling, Beverly, Mass., USA); anti-neuronal nuclei (NeuN; mouse monoclonal; 1:500; Chemicon, Temecula, Calif., USA); anti-BrdU (mouse monoclonal; 1:500; Chemicon), anti-GFP (rabbit polyclonal, 1:500; abCAM, Cambridge, Mass., USA), anti-GFAP (mouse monoclonal, 1:500; Chemicon), anti-oligodendrocyte marker O4 (anti-O4; mouse monoclonal, 1:100; Chemicon), anti-NG2 (rabbit polyclonal, 1:200; Chemicon), anti-cleaved caspase-3 (anti-caspase-3, rabbit polyclonal, 1:200; Cell Signaling). Two additional anti-DCx antibodies were evaluated (rabbit polyclonal, 1:200 abCAM, goat polyclonal, 1:50; Santa Cruz Biotechnology, Inc., Santa Cruz, Calif., USA) prior to selecting the Cell Signaling anti-DCx antibody for use in this study (online suppl. fig. 1A-C, www. karger.com/doi/10.1159/000279654). The latter two antibodies demonstrated either excessive straining (AbCam anti-DCx (online suppl. fig. 1C)) that included abundant cells coexpressing GFAP with morphologic characteristics of mature astrocytes (online suppl. fig. 1D-F) or minimal signal (Santa Cruz anti-DCx (online suppl. fig. 1A)) despite testing multiple aliquots from the company and manipulating immunohistochemistry conditions, including tissue fixation, antigen retrieval, antibody dilutions and incubation times. Secondary antibodies were purchased from Jackson laboratories (1:200; Bar Harbor, Me., USA) and Molecular Probes (1:200; Eugene, Oreg., USA). Sections were exposed to 4',6-diamidino-2-phenylindole dihydrochloride (DAPI; $1 \mu \mathrm{g} / \mathrm{ml}$; Sigma) for 1-2 min to counterstain nuclei.

\section{Cell Quantification}

Fluorescently immunolabeled sections were analyzed on a Nikon C1si Spectral Confocal Microscope (Nikon-USA, Melville, N.Y., USA). Images were acquired and reconstructed using the Nikon-EZ-C1 acquisition and analysis software, then cropped, adjusted and optimized in Photoshop 8.0 (Adobe Systems, San Jose, Calif., USA). Quantitative analysis of GFP+, GFP+/GFAP+, $\mathrm{GFP}+/ \mathrm{O} 4+, \mathrm{GFP}+/ \mathrm{NeuN}+$ cells was performed in coronal sections collected by systematic random sampling of the ipsilateral striatum at $300-\mu \mathrm{m}$ intervals. The striatum was analyzed from bregma $+2.28 \mathrm{~mm}$ to $-0.36-\mathrm{mm}$ (Plates $14-30$ Paxinos Rat Brain) and defined by the corpus callosum, superior and lateral boundaries, lateral ventricle, medial boundary and accumbens core as inferior boundary. Double-immunostained sections were imaged by randomly placing the $20 \times$ objective (Plan-Apo lens, NA 0.4 , camera resolution $1,024 \times 1,024$ field dimension $600 \times 600 \times$ $20 \mu \mathrm{m}$ ) within the striatum. Following imaging of the full thickness $\mathrm{z}$ stack (1.5- $\mu \mathrm{m}$ steps) of the $20 \times$ field, the field was manually moved a fixed distance of approximately $600 \mu \mathrm{m}$ in the horizontal and then vertical axis, resulting in 4-6 counting images per striatum per section. GFP+, and double-labeled GFP+/GFAP+, $\mathrm{GFP}+/ \mathrm{O} 4+$, GFP+/NeuN+ cells were quantified using Metamorph Offline (6.0, Universal Imaging Corporation, Downington, Pa., USA). Colocalization of double-labeled GFP+/NeuN+ was confirmed with further observation at $60 \times$ objective (PlanApo lens, NA 1.4). Cell density was calculated as average number of cells per $20 \times \mathrm{HPF}$, and cell percentage was calculated as the number of specific double-labeled cells per total number of GFP+ cells. For the olfactory bulb, quantitative analysis of GFP+ cells was performed in coronal sections collected by systematic ran- 


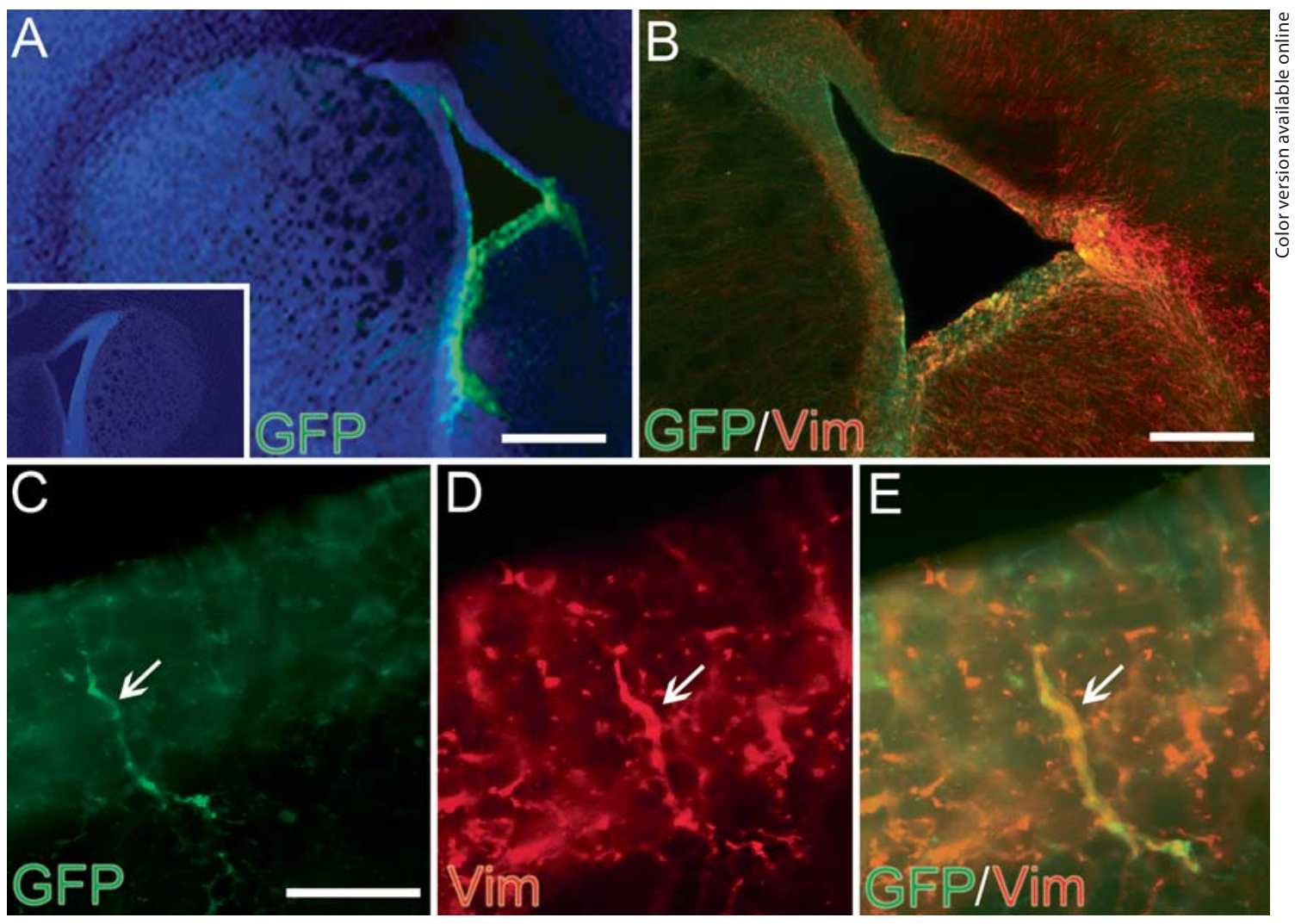

Fig. 1. Intraventricular injection of GFP-lentivirus labels SVZ NSCs. Coronal section of a $\mathrm{P} 3$ rat $48 \mathrm{~h}$ after GFP-lentiviral intraventricular injection. GFP expression (green) is primarily limited to the SVZ in the ipsilateral hemisphere (A, $4 \times$ magnification), with very few cells present in the contralateral hemisphere (A, inset, $4 \times$ ). Vimentin (Vim) immunohistochemistry (red) shows intense double labeling in the SVZ $(\mathbf{B}, 10 \times)$. At higher magnifica- tion (C-E, 60×), an example of a double-labeled GFP+ (C)/vimentin+ (D) cell with merge image (E) is seen with typical morphological features of radial glia cells (arrow), with the cell body in the SVZ and a long process moving towards the lateral ventricle. Scale bars: A $250 \mu \mathrm{m}$; B $100 \mu \mathrm{m}$; C $10 \mu \mathrm{m}$. In all figures, colors refer to the online version only. dom sampling at 600- $\mu \mathrm{m}$ intervals in the ipsilateral bulb. Sections immunostained for GFP were imaged by randomly placing the $20 \times$ objective in the olfactory bulb as described above, resulting in about 4 counting images per section.

\section{Statistical Analysis}

Results from the cell counting were analyzed for statistical significance using Student's unpaired t tests. All data are presented as means \pm standard error of the mean. Comparisons were interpreted as significant if $\mathrm{p}<0.05$.

\section{Results}

Lateral Ventricular Injection of GFP-Lentivirus Labels Ependymal and Type B Cells

In order to label cells lining the ventricle, including radial glia/type B cells, a replication incompetent lenti- virus carrying GFP driven by a ubiquitously expressed and constitutively active promoter was injected into the lateral ventricle at P1. To confirm that type B cells were labeled, we examined GFP expression in the SVZ, RMS, ipsilateral olfactory bulb and striatum. Two days after the injections, intense GFP expression was observed and was limited to the ipsilateral lateral ventricle and SVZ (fig. 1A), with labeled cells visible from the rostral to caudal regions of the forebrain. GFP+ cells were rarely observed in the contralateral ventricle (fig. 1A, inset). Several GFP+ cells with processes typical of radial glia, extending from the cell body towards the ventricular surface, were detected (fig. 1C). These cells also expressed the radial glia marker vimentin, a member of the intermediate filament family (fig. 1B, D, E). Ependymal cells lining the lateral ventricle are also labeled (fig. 1C). 

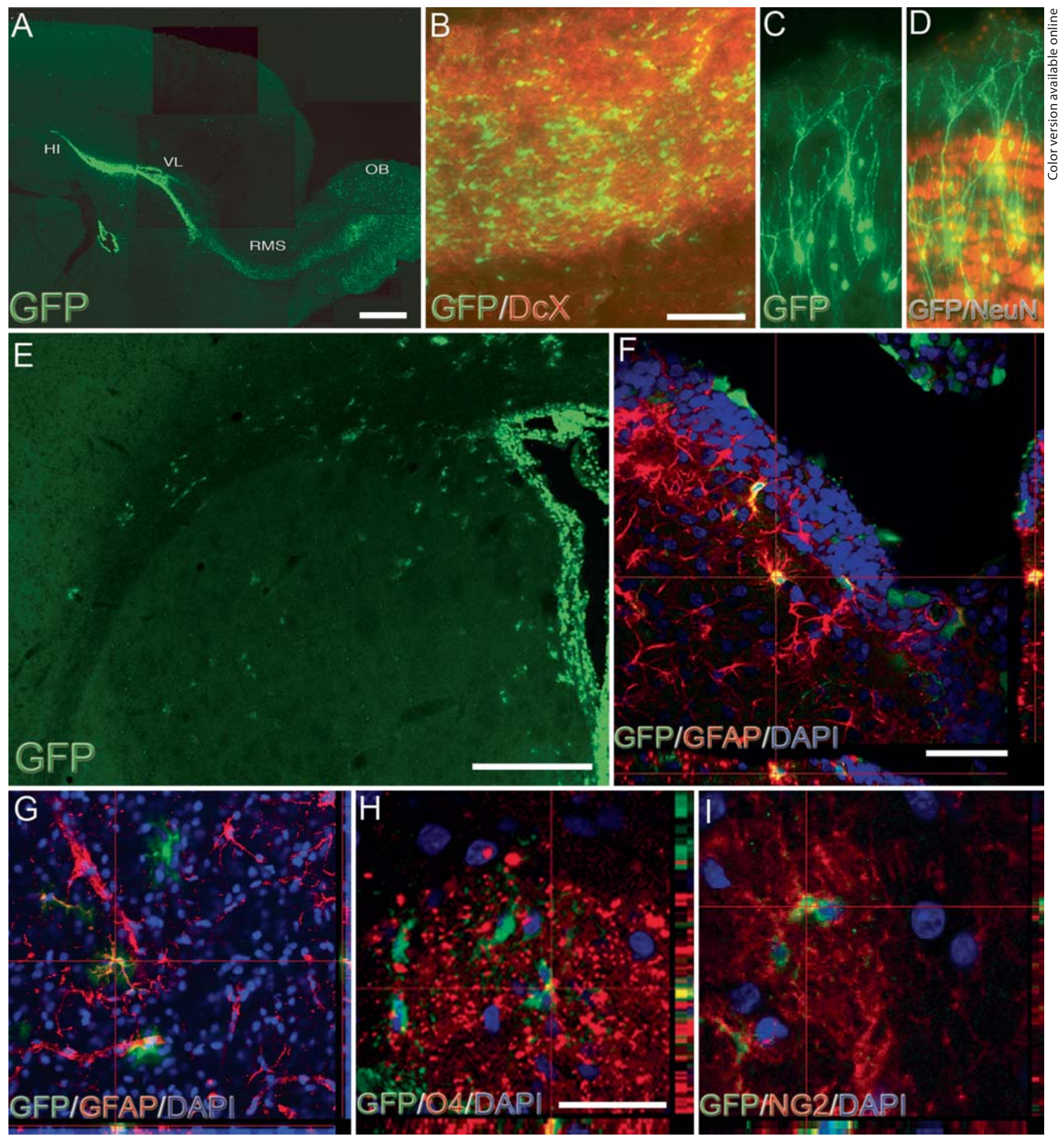

Fig. 2. Progeny of GFP-lentiviral-labeled NSCs. Analysis of a P21 rat forebrain 3 weeks after intraventricular injection. Sagittal section demonstrates a tangential migration pattern of GFP+ cells (green) labeled in the SVZ at P1 (A, $4 \times$ magnification). These cells coexpress DCx (red), a marker of immature neurons (B, 20X), which join the RMS. In the olfactory bulb (OB) cortex, GFP+ cells $(C, 60 \times)$ with mature neuronal morphology express NeuN (D, red). GFP is stably expressed in the ipsilateral SVZ $(\mathbf{E}, 10 \times)$, with a coro- nal section showing the fate of cells radially migrating from the SVZ towards the overlying structures. Colabeled GFAP+ astrocytes (red) are seen at the SVZ/striatal boundary (F, 40X), and in the striatum (G). DAPI (blue) is used as a nuclear counterstain. Double labeling of $\mathrm{O} 4+(\mathbf{H})$ and $\mathrm{NG} 2+(\mathbf{I})$ in the striatum is also seen. Scale bars: $\mathbf{A} 250 \mu \mathrm{m}$; $\mathbf{B} 50 \mu \mathrm{m}$; E $250 \mu \mathrm{m}$; F, G $25 \mu \mathrm{m}$; H, I $10 \mu \mathrm{m}$. HI = Hippocampus; $\mathrm{VL}=$ lateral ventricle. 
Neonatal Lentiviral Injections Result in GFP-Labeled Olfactory Neurons and Striatal Glia

At P21, expression of GFP+ cells persists in the SVZ (fig. 2A, E). A tangential stream of GFP+ cells was observed covering virtually the entire rostrocaudal extent of the SVZ, the RMS and olfactory bulb (fig. 2A). The RMS contained many cells positive for both GFP and $\mathrm{DCx}$, a microtubule-associated protein expressed primarily by migrating immature neurons (fig. 2B). Cells were also observed in granular and periglomerular layers of the olfactory bulb cortex (fig. 2C), where they expressed NeuN, a marker of mature neurons (fig. 2D).

Some cells were located between the SVZ and the striatum with the 'bushy' characteristics of astrocytes (fig. 2E, F). These cells express GFAP (fig. 2F). As previously reported [12, 13, 24], GFP-labeled cells migrated radially towards the overlying structures, including the striatum, white matter and neocortex. Most of these cells expressed either GFAP (fig. 2G), O4 (fig. 2H) or NG2 (fig. 2I) at P21, markers of mature astrocytes and immature oligodendrocytes, respectively. No cells were identified in the striatum that expressed DCx.

\section{Neonatal MCAO Injures the Striatum or Striatum and Neocortex}

Animals were selected for this study based upon DWRMI demonstrating a mild injury pattern with hyperintense signal (restricted diffusion) limited to subcortical structures (fig. $3 \mathrm{~A}, \mathrm{n}=4$ ) or moderate/severe injury with both subcortical and cortical injury (fig. 3D, $\mathrm{n}=2$ ). Histolologic assessment at P21 confirmed this pattern. The injury is characterized by: neuronal loss (fig. 3B) and intense astrocy tosis (fig. 3C) localized in the ipsilateral striatum. Contralateral structures (not shown) showed no injury. In comparison, moderate injury included cortical structures with increased diffusion on MRI (fig. 3D), columnar cortical neuronal loss (fig. 3E) and astrocytosis (fig. 3F). In animals with both mild and moderate injury, GFP-expressing cells are observed moving away from the SVZ toward the striatum (fig. 3B, E, insets). Animals with moderate injury show comparatively less GFP-expressing cells.

\section{No GFP-Labeled Striatal Neurons Can Be Identified following $M C A O$}

Recent evidence has shown that the SVZ may be the source of newly generated striatal neurons following hypoxia-ischemia $[5,23]$ or stroke $[1,2]$ in both neonatal and adult rodents. To detect immature neurons, immunohistochemistry for DCx was performed at both P14 $(\mathrm{n}=2)$ and P21 $(\mathrm{n}=6)$. In animals with both mild $(\mathrm{n}=6)$ and moderate $(\mathrm{n}=2)$ injury, DCx expression increased in the SVZ (fig. 4A). A limited number of DCx+ cells were observed at the boundary between the white matter and the striatum (fig. 4A); however, in no cases were cells observed that coexpress GFP and DCx $(n=8)$. In order to detect mature neurons originating from the SVZ NSC lineage following stroke, GFP+/NeuN+ double labeling was performed. In neither the injured nor the sham groups were $\mathrm{GFP}+/ \mathrm{NeuN}+$ cells detected after $\mathrm{MCAO}$ $(\mathrm{n}=7)$.

\section{MCAO Reduces the Neurogenic Activity of SVZ NSC Lineage}

Twenty-four hours after MCAO, many GFP+ cells in the SVZ coexpress cleaved caspase-3, an apoptotic marker, suggesting cell death among progenitor cells soon after transient ischemic stroke (fig. 4B, arrows). Most of these cells are localized within SVZ boundaries. Consistent with this finding of increased cell death in the progenitor population, expression of GFP decreased in the SVZ in animals treated with MCAO compared with controls (fig. 4C, D). Marked reduction of BrdU expression was also observed in the SVZ in MCAO animals compared to sham-treated animals (fig. 4E, F). The density of GFP+ cells in the olfactory bulb at 2 weeks after MCAO was then determined as an additional marker of neurogenic activity in this lineage (fig. 4G, H). Sham-treated animals had $58.8 \pm 14.9 \mathrm{GFP}+$ cells/HPF in the ipsilateral olfactory bulb, while injured animals had a density of $19.6 \pm 5.4 / \mathrm{HPF}(\mathrm{p}=0.025)$ (fig. $4 \mathrm{I})$.

\section{SVZ-Derived Striatal Gliogenesis Is Reduced following} MCAO, with an Increased Astrocyte/Oligodendrocyte

Ratio

To examine the effect of MCAO on SVZ NSC progeny in the striatum, the density of GFP+ cells was determined at P21. In the injured striatum, the majority of GFP+ cells were located close to the ventricle, with a few cells in the central part of the structure and none in a region close to the subcortical white matter at 2 weeks after MCAO (fig. 5A, B). Consistent with observations in the olfactory bulb, quantification of GFP+ cells in the striatum showed a significant decrease in cells in injured animals compared to sham controls (compare fig. 2E with fig. 5A, 12.7 \pm 7.3 vs. $2.8 \pm 0.6$ cells/HPF, $p=0.01$, fig. $5 \mathrm{C}$ ). Double labeling for GFP+/GFAP+ and GFP+/O4+ (fig. 2G, H) was also quantified in the striatum and showed a significant reduction of both astrocytes $(4.8 \pm 1.02$ vs. $2.5 \pm$ 0.4 cells/HPF; $\mathrm{p}=0.005)$ and oligodendrocytes $(2.8 \pm 1$ 

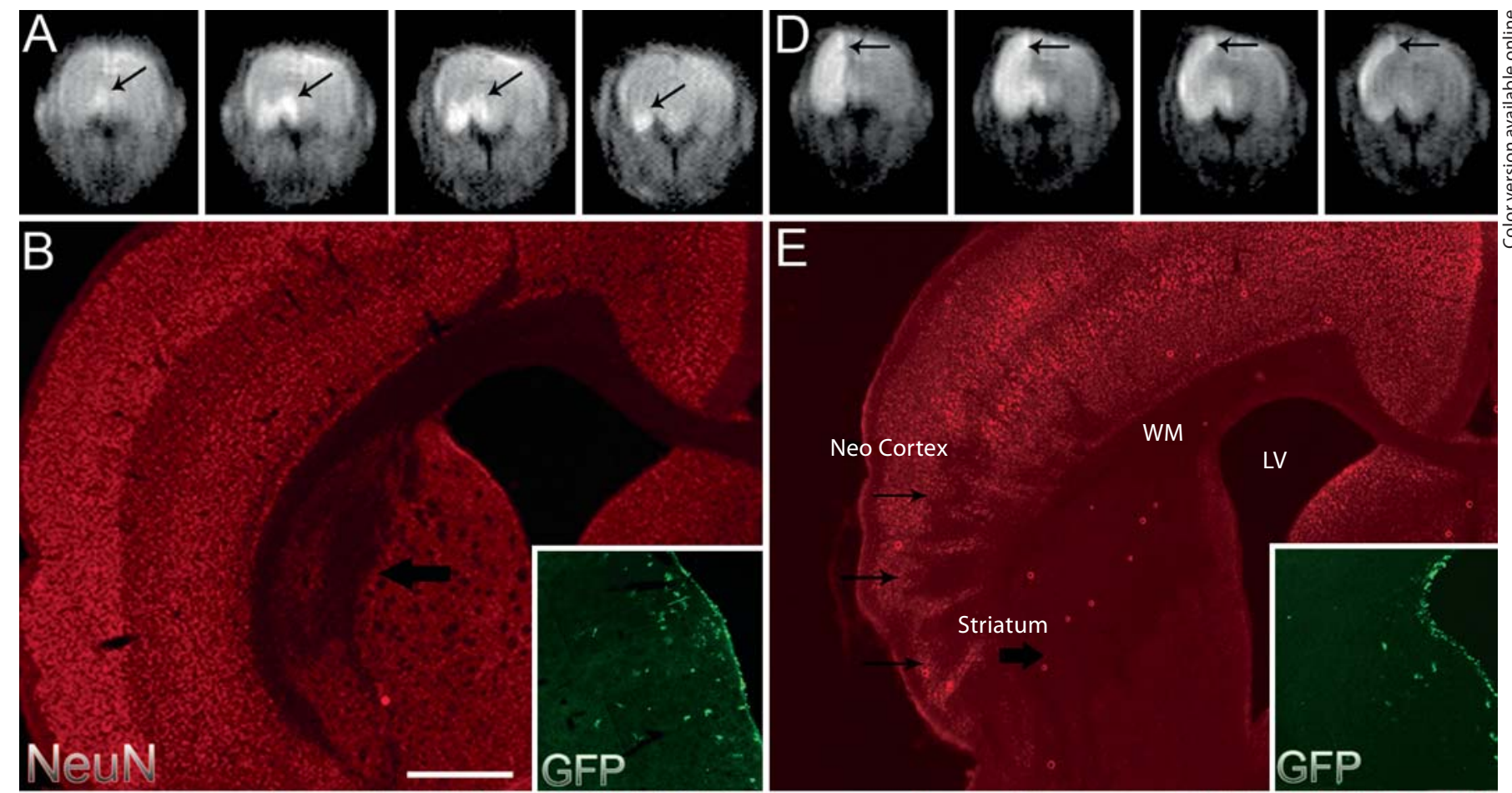

Neo Cortex

WM
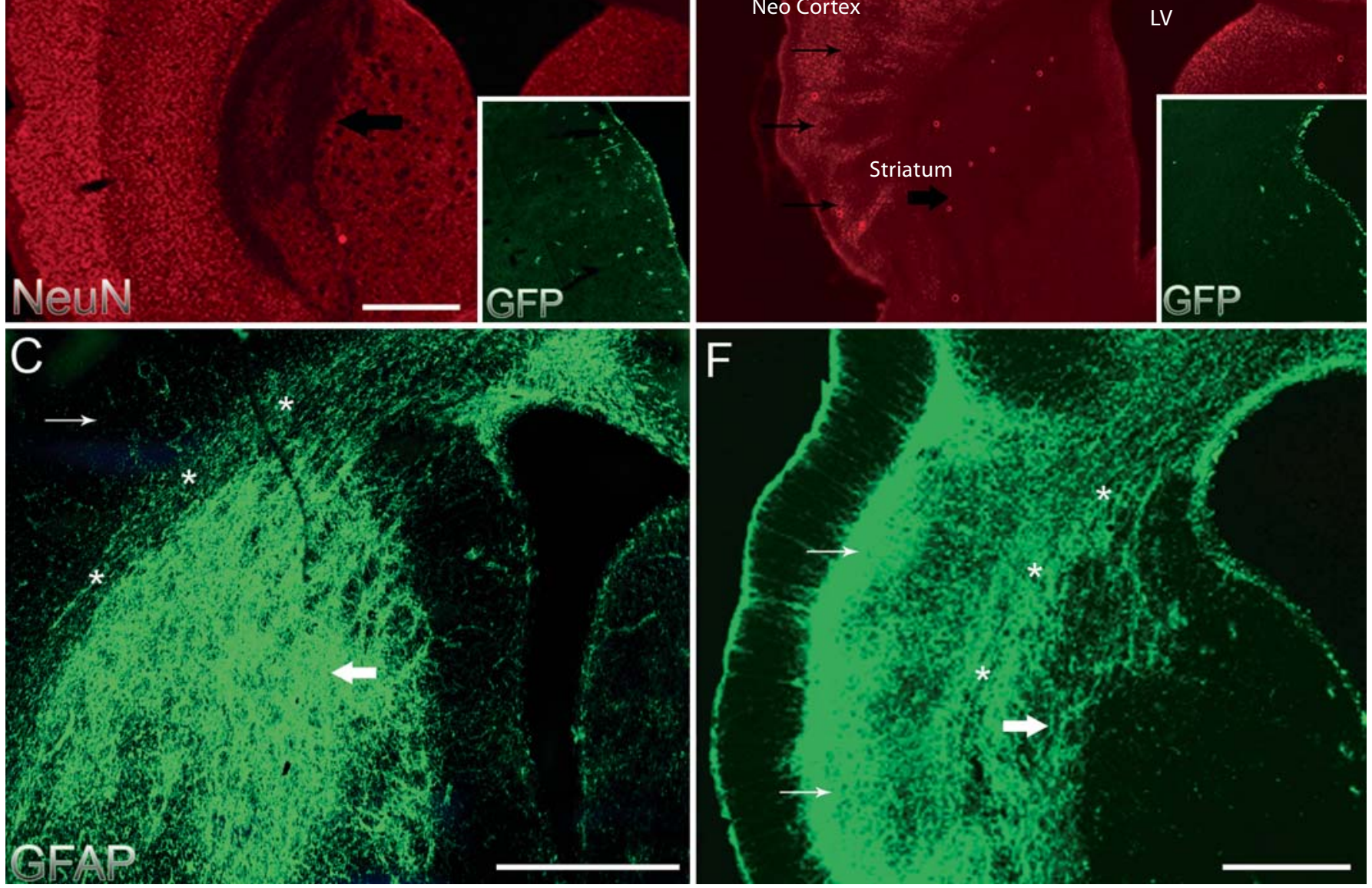

Fig. 3. Histopathology of MCAO leading to mild or severe injury. Anterior to posterior coronal image slices of DW-MRI performed during occlusion demonstrates an ipsilateral subcortical lesion (arrows) in mild injury (A) whereas more diffuse cortical injury is seen in severe injury (D). In coronal sections of P21 rat brain, the ipsilateral hemisphere shows significant reduction (arrow) of neurons (NeuN, red) in the striatum 2 weeks after MCAO that resulted in mild injury (B). GFP+ cells in ipsilateral SVZ and medial striatum in mild injury is also shown (B, inset, 20×). GFAP+ astrocytic expression (green) is localized to the striatum (large

arrow) in mild injury, with minimal staining noted in cortical region (small arrow) distal to white matter (asterisks) $(\mathbf{C}, 10 \times)$. In severe injury, more diffuse loss of $\mathrm{NeuN}+$ neurons in ipsilateral striatum (large arrow) and cortex (small arrows) is noted (E, $4 \times$ ), with fewer GFP+ cells (E, inset, $20 \times)$. There is also an increase in GFAP+ staining in cortex (small arrows), indicative of more severe injury $(\mathbf{F}, 4 \times)$. Asterisks indicate region of cortical white matter. Scale bars: $250 \mu \mathrm{m}$. WM = White matter; $L V=$ lateral ventricle. 

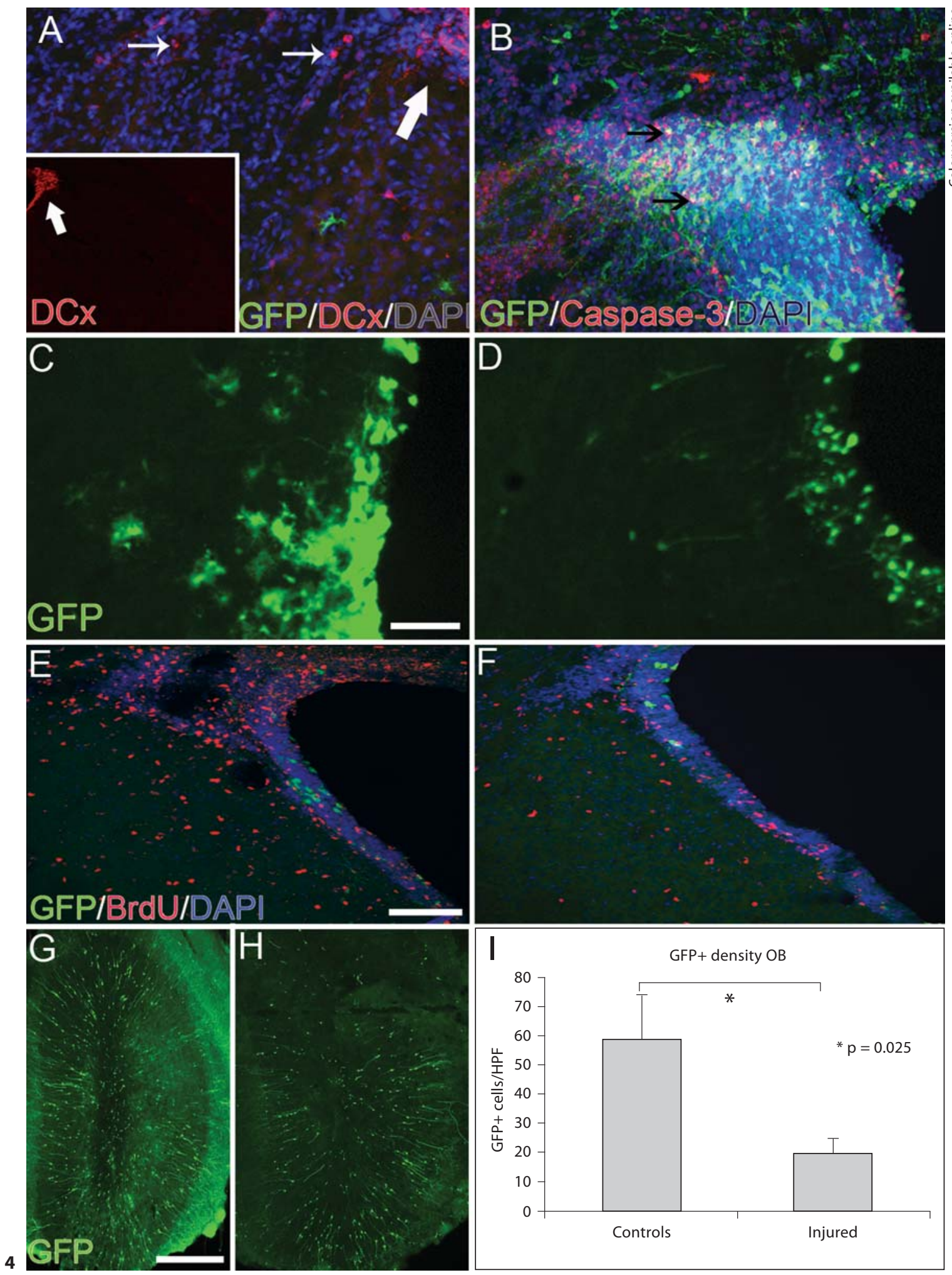
Fig. 5. MCAO effects on postnatal SVZderived glial cells in the striatum. MCAO induces marked astrogliosis (GFAP, red) in ipsilateral striatum $(\mathbf{A}, 10 \times)$. Higher magnification of colabeled cells is also shown $(B, 20 \times)$. Quantification of GFP+ cells in the striatum showed a significant $(\mathrm{p}=0.001)$ decrease in cells in MCAO brains compared to controls (C). The densities of GFP+/GFAP+ astrocytes and $\mathrm{GFP}+/ \mathrm{O} 4+$ oligodendrocytes were both significantly reduced in injured animals ( $\mathrm{p}=0.005$ and 0.008 , respectively) (D). Scale bar: A $250 \mu \mathrm{m}$.
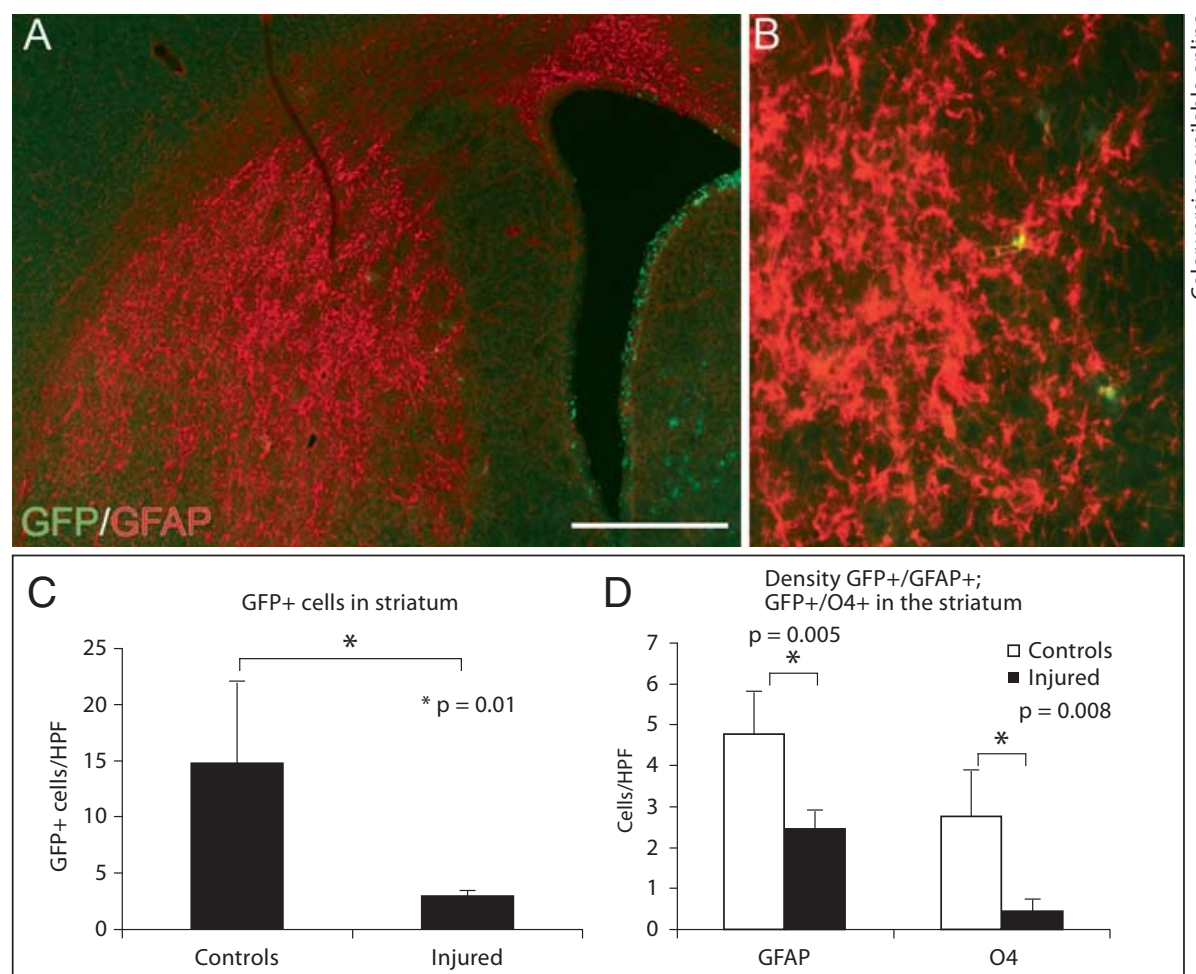

D

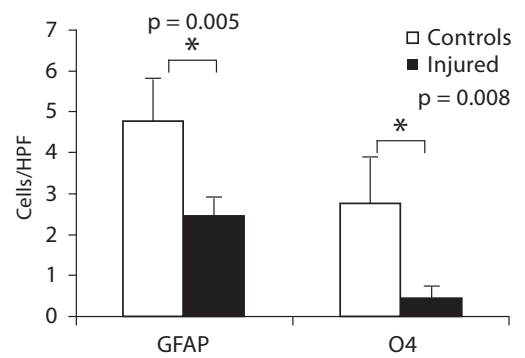

vs. $0.5 \pm 0.25 ; \mathrm{p}=0.008$ ) (fig. $5 \mathrm{D}$ ). In sham-treated animals, $40 \%$ of GFP+ cells were colabeled with GFAP, while $30 \%$ were colabeled with $\mathrm{O} 4$. In MCAO animals, there was an increase in the proportion of colabeled astrocytes relative to oligodendrocytes, with $70 \%$ of GFP+ cells expressing GFAP and $14 \% \mathrm{O} 4$. Interestingly, the percentage of GFP+ cells that were GFAP-/O4- remained relatively unchanged.

Fig. 4. MCAO alters neurogenesis of GFP-lentiviral-labeled NSCs. Increased expression of $\mathrm{DCx}+$ cells $(\mathbf{A}, \mathrm{red})$ in the ipsilateral SVZ (large arrow), white matter (small arrows) and striatum relative to contralateral side at P21 $(\mathbf{A}$, inset, $10 \times$; large arrow $=\mathrm{SVZ})$ and sham-treated animals (not shown). These DCX+ cells did not coexpress GFP (green). At P8 (24 h after MCAO), increased expression of cleaved caspase-3 (red) is seen in the ipsilateral SVZ, some of which coexpress GFP (black arrows) (B, 20×). At P21, GFP expression is shown in ipsilateral SVZ of a control animal $(\mathbf{C}, 40 \times)$, with decreased GFP expression in MCAO animals (D). Detection of BrdU+ cells (red) in SVZ of control (E) and MCAO (F) brain shows marked reduction after injury. GFP+ cells are shown in the olfactory bulb in a control $(\mathbf{G})$ and MCAO animal $(\mathbf{H})$ at P21. Quantification of GFP+ cells in the olfactory bulb (OB) shows a significant $(\mathrm{p}=0.025)$ reduction in MCAO versus control animals (I). Scale bars: C $100 \mu \mathrm{m}$; E, G $250 \mu \mathrm{m}$.

\section{Discussion}

This study shows for the first time that NSCs associated with the lateral ventricle, including type B cells and/ or ependymal cells, do not significantly contribute to neurogenesis following neonatal stroke and in fact, are among the cell populations adversely affected by the injury. After efficient and stable labeling of these SVZ NSC lineages with a lentivirus carrying GFP, no cells coexpressing GFP and either DCx or NeuN, markers of immature and mature neurons respectively, were detected in the injured striatum after injury. Some DCx+ cells were detected near the ischemic lesion in the striatum, but did not coexpress GFP. Although newborn cells originating from type B/radial glia SVZ NSCs do not include striatal neurons following neonatal transient ischemic stroke, this lineage may participate in repair through astrogliogenesis. However, even among this population, cells originating from the type $\mathrm{B} /$ radial glial lineage are a minor contributor to the massive gliogenesis observed following neonatal stroke as evidenced by small numbers of GFP-labeled astrocytes despite extensive numbers of cells expressing GFAP. The findings of this study also demonstrate that an acute ischemic event disrupts post- 
natal SVZ-derived glia in the striatum, resulting in a global depletion of glia cells and an increase in the ratio of astrocytes to oligodendrocytes among the survivors.

In this study, we used a highly efficient GFP-lentivirus that infects SVZ NSCs when administered via intraventricular injection, by infecting both rapidly dividing and slowly dividing or nondividing cells [19]. A number of lines of evidence confirm that we have labeled type $\mathrm{B}$ NSCs. First, GFP+ cells appear in the SVZ 2 days after lentivirus injections with radial glia morphology. These GFP-labeled cells coexpress vimentin, a marker of type B cells. Second, 2 weeks after the injections, abundant $\mathrm{GFP}+/ \mathrm{DCx}+$ cells are detected in the RMS. Third, 4 weeks after the injection, abundant GFP+/NeuN+ cells with neuronal morphology are noted in the olfactory bulb cortex. Lastly, intraventricular injection results in labeled striatal glia including astrocytes, oligodendrocytes and NG2-positive cells. Thus, intraventricular injection of GFP-lentivirus robustly labels all cells known to emerge from the SVZ type B cell lineage. These injections also label ependymal cells. Although we did not attempt to specifically identify them, we expect that labeled type C, transit-amplifying cells will inherit the GFP label from transduced type B cells.

A major observation in this study is that no GFP-labeled striatal or cortical neurons were observed following MCAO. Consistent with prior observations [25], we did observe some DCx-expressing immature neurons in striatum, but none expressed GFP. Two possible conclusions can explain these results: (1) These newborn neurons arise from some other lineage than SVZ type B-derived cells. (2) Intraventricular GFP-lentiviral transduction of type B cells is inefficient, labeling only a small percentage of the stem cell pool. Estimating the percentage of labeled NSCs in the total pool of type B cells is difficult. It is possible that some NSCs may not be infected; however, the homogeneous distribution of the virus by the intraventricular approach, as well as the abundant labeling of olfactory bulb neurons suggests that this technique marks a substantial fraction of the stem cell pool. We failed to observe any DCx- or NeuN-GFP-labeled cells in the striatum in multiple animals with both mild and moderate injuries. Consistent with our findings is a report utilizing a recently developed approach based upon a nestinCreER system in mice to label SVZ NSCs and SVZ-derived newly generated neurons [26]. Although this experiment was performed in adult mice and therefore may not be directly comparable to the present experiments in neonatal rats, this approach also failed to identify any cells expressing DCx derived from SVZ NSCs after an acute ischemic event. The authors concluded that there was very little contribution of cell replacement by the SVZ NSCs following focal cerebral ischemia in the adult brain. Furthermore, the authors compared the results obtained with the Cre system to BrdU labeling of newly generated cells, which showed that most BrdU+ cells were not derived from SVZ progenitors.

Another important finding is that SVZ type B cell lineage has significantly reduced overall neurogenic activity as evidenced by a significant reduction in SVZ-derived neurons in the olfactory bulb 2 weeks after injury. Reduced olfactory neurogenesis occurs despite an increase in SVZ DCx expression, suggesting either a block in migration and/or persistently increased progenitor cell death. Previous studies have shown consistent depletion of progenitor cells in SVZ following severe hypoxia-ischemia $[27,28]$. Cleaved caspase-3, a marker of apoptosis, was also detected as early as $4 \mathrm{~h}$ after reperfusion and peaked at $24 \mathrm{~h}$ after the insult, coinciding with our findings. More importantly, at 3 weeks after injury, the SVZ was smaller and markedly less cellular, and contained less than a quarter of the normal complement of NSCs compared to sham-treated animals, confirming that NSCs are vulnerable to hypoxia-ischemia [27].

We observed a reduction of BrdU expression in the SVZ in injured animals compared to controls at P21. This is consistent with the lentivirus findings that show a marked reduction of GFP+ cells in the injured SVZ compared to controls. The literature offers contrasting data regarding BrdU-labeled cells in the SVZ in the first 2-3 weeks following the injury with reports of an increased $[1,2,4]$, unchanged $[29,30]$ or decreased amount of labeled cells [27]. Data differ depending on the age of the animal, the severity of the insult and the ischemia model used. Since hypoxia alone is able to increase BrdU expression in SVZ, this may influence the results [29]. Finally, concerns regarding the use of BrdU have been raised, since label may be taken up without cells dividing [31] and, more importantly, damaged neurons in ischemic brain regions may re-enter the cell cycle in a process of abortive DNA synthesis prior to dying via apoptosis [32]. For example, hypoxia-ischemia in adult rodents has been reported to induce neurons to resume DNA synthesis and incorporate BrdU [32].

Recently, sustained neuronal replacement has been detected in the rodent forebrain after a severe cortical and striatal infarction in response to neonatal (P6) hypoxiaischemia [23]. In this model, the SVZ was identified as source of newly generated cells by labeling stem and/or progenitor cells through intraventricular injection of a 
retrovirus carrying human placental alkaline phosphatase $24 \mathrm{~h}$ before the injury (P5). Several possibilities relating to differences in the models, injury severity and assessment of neurogenesis may explain the divergent observations between the present results and the findings from this prior study. Regarding the models, hypoxiaischemia may be a more potent stimulus for neurogenesis by engaging hypoxia-related signaling pathways including HIF-1a, VEGF and erythropoietin [33, 34]. A second issue relates to injury severity and location. As reviewed above, the neonatal SVZ is uniquely susceptible to damage [27]. Despite this finding, robust cortical neurogenesis has been observed following neonatal hypoxia-ischemia with moderate to severe injury $[23,27]$. The present study is the first to consider these issues in a neonatal focal ischemia and reperfusion model. Findings are consistent with regard to cell death in the SVZ. However, reduced neurogenic activity is observed in all SVZ NSC progeny following neonatal MCAO including olfactory neurons and striatal glia. The lack of cortical neurogenesis may relate to a less severe cortical injury following MCAO (fig. 3D-F) compared with neonatal hypoxiaischemia [23]. Lastly, differences between the studies may have resulted from the techniques used to identify SVZ stem and progenitor cells: retrovirus compared with lentivirus and specific antibodies against DCx. Regarding viral transduction, retroviruses are only capable of infecting dividing cells, and have shown poor efficiency in labeling slowly dividing NSCs, while lentivirus will label both populations [19]. Although we expect that all cells in the lineage produced by SVZ type B cells should inherit the lentiviral-delivered GFP label, including transitamplifying cells, retroviral transduction may target intermediate progenitors more efficiently, resulting in differences in the final labeled populations. Finally, we observed significant variability testing multiple antiDCx antibodies and staining conditions (online suppl. fig. 1) with one antibody giving abundant, nonspecific signal that labeled both neuroblasts and astrocytes, while another commonly used antibody labeled only rare cells despite extensive manipulation of experimental conditions. These observations encourage caution when interpreting results identifying newborn neurons based solely upon DCx expression and may explain differences in the magnitude of striatal and cortical neurogenesis observed in the present study compared with prior studies.

The presence of newly generated immature neurons that were not labeled by ventricular lentiviral-GFP injections suggests other progenitor populations may be involved in the neuroregenerative response following stroke. This would include the subcallosal zone (SCZ), a lamina of dividing cells that corresponds to the caudal and medial extension of the posterior horn of the lateral ventricle, and can be considered a caudal and dorsomedial extension of the SVZ [35]. The SCZ is not associated with an overt opening to the ventricular cavity, so its cells should not be labeled with a GFP-lentivirus administered intraventricularly [35]. The SCZ has been shown to contain NSCs in vitro, and to produce astrocytes and oligodendrocytes in vivo [35]. There is SCZ proliferation following kainate-induced seizures [36]. Following neonatal stroke, newly generated neurons may be produced in the SCZ, moving tangentially along the lateral ventricle within the SVZ and then migrating radially, or through the subcortical white matter, to reach the injured area. Resident NSCs in the neocortex may also be an alternative source of newly generated cells. A recent report described the presence of NG2+ cortical cell populations that express nestin following adult stroke, and had the capacity for self-renewal and differentiation into electrophysiologically functional neurons, astrocytes and myelin producing oligodendrocytes in vitro [37].

We observed that neonatal stroke dramatically reduced the overall number of postnatal SVZ-derived glial cells in the striatum and shifted the percentage of glia cells toward astrocytes. Although we did not quantify doublelabeled NG2/GFP+ cells, this population likely accounts for the O4-/GFAP- cells ( 26\%). Using a different method to identify newborn glia (nestin-CreER), Burns et al. [38] observed a percentage of labeled astrocytes and oligodendrocytes, similar to that in the present results. In their study, the remaining labeled cells were described as simple glioblasts or polydendritic glioblasts expressing NG2. Furthermore, this study combined BrdU labeling with genetic fate mapping to determine that cortical GFAP-expressing astrocytes were largely postmitotic [38]. In comparison, about $10 \%$ of NG2-expressing cells remained proliferative in the postnatal brain and this percentage increased following brain injury [38]. Proliferation of NG2 cells has been observed in cortex following neonatal hypoxia-ischemia $[39,40]$. NG2 cells generate astrocytes in spinal cord both normally and following injury [41, 42], suggesting that this population may be a source of the postinjury proliferative gliosis in striatum and cortex. The shift we observed favoring astrocytes could represent selective loss of $\mathrm{O} 4+$ oligodendrocyte progenitors or proliferation of this NG2 population with astrocytic differentiation. Depletion of O4+ cells, a marker of preoligodendrocytes, has been observed in every region of the neonatal brain following hypoxia-ischemia, includ- 
ing the cortex [43], white matter [40] and the striatum [43]. These findings represent death of a specifically vulnerable progenitor population. Unfortunately, recent evidence suggests that these proliferating cells may not become myelinating oligodendrocytes due to an arrest of maturation following the injury [40]. Our data showed a marked reduction of postnatal SVZ derived O4+ cells following stroke and no sign of proliferation despite the presence of some SVZ-derived NG2+ cells.

Interestingly, among the surviving glial cells in the striatum we detected an increase in the ratio of astrocytes to oligodendrocytes. Although this may be due to loss of preoligodendrocytes, this finding may also be explained by a shift in cell fate choice of SVZ glial progenitors toward astrocytes following stroke. Recently, it has been shown that a single administration of erythropoietin in the reperfusion phase significantly reduces the number of newly generated cells expressing GFAP after ischemic insult and increases the percentage of newly generated neurons [22]. Given the presence of erythropoietin receptors on radial glia cells, it may be possible that growth factor effects influence the commitment of these newly generated cells.

Our data focus for the first time on specific stem cell lineages associated with the ventricle in a stroke model of developing brain. These include SVZ type B NSCs, suggesting that this cell population may contribute to newly generated neurons after injury. Additional studies are necessary to confirm these findings but new efforts should be devoted to define the presence of alternative sources of new cells in the developing forebrain. Furthermore, the implications for repair and the role of gray matter glial development after neonatal stroke needs to be further explored.

\section{Acknowledgments}

This study was supported by March of Dimes and ASA/Bugher grants. The authors would like to thank Vien Nguyen and the Pediatric Developmental Biology Laboratory for technical support. Data for this study were acquired at the Nikon Imaging Center at UCSF.

\section{References}

-1 Arvidsson A, Collin T, Kirik D, Kokaia Z, Lindvall O: Neuronal replacement from endogenous precursors in the adult brain after stroke. Nat Med 2002;8:963-970.

-2 Parent JM, Vexler ZS, Gong C, Derugin N, Ferriero DM: Rat forebrain neurogenesis and striatal neuron replacement after focal stroke. Ann Neurol 2002;52:802-813.

-3 Yang Z, Levison SW: Hypoxia/ischemia expands the regenerative capacity of progenitors in the perinatal subventricular zone. Neuroscience 2006;139:555-564.

4 Plane JM, Liu R, Wang TW, Silverstein FS, Parent JM: Neonatal hypoxic-ischemic injury increases forebrain subventricular zone neurogenesis in the mouse. Neurobiol Dis 2004;16:585-595

5 Ong J, Plane JM, Parent JM, Silverstein FS: Hypoxic-ischemic injury stimulates subventricular zone proliferation and neurogenesis in the neonatal rat. Pediatr Res 2005;58:600606.

6 Ackman JB, Siddiqi F, Walikonis RS, LoTurco JJ: Fusion of microglia with pyramidal neurons after retroviral infection. J Neurosci 2006;26:11413-11422.

7 Kuhn HG, Cooper-Kuhn CM: Bromodeoxyuridine and the detection of neurogenesis. Curr Pharm Biotechnol 2007;8:127-131.

-8 Taupin P: BrdU immunohistochemistry for studying adult neurogenesis: Paradigms, pitfalls, limitations, and validation. Brain Res Rev 2007;53:198-214.
-9 Sergent-Tanguy S, Michel DC, Neveu I, Naveilhan P: Long-lasting coexpression of nestin and glial fibrillary acidic protein in primary cultures of astroglial cells with a major participation of nestin(+)/GFAP(-) cells in cell proliferation. J Neurosci Res 2006;83:1515-1524.

10 Lois C, Alvarez-Buylla A: Proliferating subventricular zone cells in the adult mammalian forebrain can differentiate into neurons and glia. Proc Natl Acad Sci USA 1993;90: 2074-2077.

11 Doetsch F, Alvarez-Buylla A: Network of tangential pathways for neuronal migration in adult mammalian brain. Proc Natl Acad Sci USA 1996;93:14895-14900.

-12 Levison SW, Goldman JE: Both oligodendrocytes and astrocytes develop from progenitors in the subventricular zone of postnatal rat forebrain. Neuron 1993;10:201-212.

13 Suzuki SO, Goldman JE: Multiple cell populations in the early postnatal subventricular zone take distinct migratory pathways: a dynamic study of glial and neuronal progenitor migration. J Neurosci 2003;23:4240-4250.

14 Doetsch F, Caille I, Lim DA, Garcia-Verdugo JM, Alvarez-Buylla A: Subventricular zone astrocytes are neural stem cells in the adult mammalian brain. Cell 1999;97:703-716.
15 Merkle FT, Tramontin AD, Garcia-Verdugo JM, Alvarez-Buylla A: Radial glia give rise to adult neural stem cells in the subventricular zone. Proc Natl Acad Sci USA 2004;101: 17528-17532.

-16 Mirzadeh Z, Merkle FT, Soriano-Navarro M, Garcia-Verdugo JM, Alvarez-Buylla A: Neural stem cells confer unique pinwheel architecture to the ventricular surface in neurogenic regions of the adult brain. Cell Stem Cell 2008;3:265-278.

-17 Merkle FT, Alvarez-Buylla A: Neural stem cells in mammalian development. Curr Opin Cell Biol 2006;18:704-709.

18 Lois C, Hong EJ, Pease S, Brown EJ, Baltimore D: Germline transmission and tissuespecific expression of transgenes delivered by lentiviral vectors. Science 2002;295:868872 .

19 Geraerts M, Eggermont K, Hernandez-Acosta P, Garcia-Verdugo JM, Baekelandt V, Debyser Z: Lentiviral vectors mediate efficient and stable gene transfer in adult neural stem cells in vivo. Hum Gene Ther 2006;17:635650

20 Carlen M, Meletis K, Goritz C, Darsalia V, Evergren E, Tanigaki K, Amendola M, Barnabe-Heider F, Yeung MS, Naldini L, Honjo T, Kokaia Z, Shupliakov O, Cassidy RM, Lindvall O, Frisen J: Forebrain ependymal cells are notch-dependent and generate neuroblasts and astrocytes after stroke. Nat Neurosci 2009;12:259-267. 
-21 Derugin N, Ferriero DM, Vexler ZS: Neonatal reversible focal cerebral ischemia: a new model. Neurosci Res 1998;32:349-353.

-22 Gonzalez FF, McQuillen P, Mu D, Chang Y, Wendland M, Vexler Z, Ferriero DM: Erythropoietin enhances long-term neuroprotection and neurogenesis in neonatal stroke. Dev Neurosci 2007;29:321-330.

-23 Yang Z, Covey MV, Bitel CL, Ni L, Jonakait GM, Levison SW: Sustained neocortical neurogenesis after neonatal hypoxic/ischemic injury. Ann Neurol 2007;61:199-208.

-24 Zerlin M, Levison SW, Goldman JE: Early patterns of migration, morphogenesis, and intermediate filament expression of subventricular zone cells in the postnatal rat forebrain. J Neurosci 1995; 15:7238-7249.

-25 Chang YS, Mu D, Wendland M, Sheldon RA, Vexler ZS, McQuillen PS, Ferriero DM: Erythropoietin improves functional and histological outcome in neonatal stroke. Pediatr Res 2005;58:106-111.

-26 Burns KA, Ayoub AE, Breunig JJ, Adhami F, Weng WL, Colbert MC, Rakic P, Kuan CY: Nestin-creer mice reveal DNA synthesis by nonapoptotic neurons following cerebral ischemia hypoxia. Cereb Cortex 2007;17: 2585-2592.

-27 Levison SW, Rothstein RP, Romanko MJ, Snyder MJ, Meyers RL, Vannucci SJ: Hypoxia/ischemia depletes the rat perinatal subventricular zone of oligodendrocyte progenitors and neural stem cells. Dev Neurosci 2001;23:234-247.

-28 Romanko MJ, Zhu C, Bahr BA, Blomgren K, Levison SW: Death effector activation in the subventricular zone subsequent to perinatal hypoxia/ischemia. J Neurochem 2007;103: 1121-1131.
-29 Iwai M, Ikeda T, Hayashi T, Sato K, Nagata T, Nagano I, Shoji M, Ikenoue T, Abe K: Temporal profile of neural stem cell proliferation in the subventricular zone after ischemia/ hypoxia in the neonatal rat brain. Neurol Res 2006;28:461-468.

30 Kadam SD, Mulholland JD, McDonald JW, Comi AM: Poststroke subgranular and rostral subventricular zone proliferation in a mouse model of neonatal stroke. J Neurosci Res 2009;87:2653-2666.

>31 Cooper-Kuhn CM, Kuhn HG: Is it all DNA repair? Methodological considerations for detecting neurogenesis in the adult brain. Brain Res Dev Brain Res 2002;134:13-21.

32 Kuan CY, Schloemer AJ, Lu A, Burns KA, Weng WL, Williams MT, Strauss KI, Vorhees CV, Flavell RA, Davis RJ, Sharp FR, Rakic P. Hypoxia-ischemia induces DNA synthesis without cell proliferation in dying neurons in adult rodent brain. J Neurosci 2004;24: 10763-10772.

33 Iwai M, Cao G, Yin W, Stetler RA, Liu J, Chen J: Erythropoietin promotes neuronal replacement through revascularization and neurogenesis after neonatal hypoxia/ischemia in rats. Stroke 2007;38:2795-2803.

34 Chen ZY, Asavaritikrai P, Prchal JT, Noguchi CT: Endogenous erythropoietin signaling is required for normal neural progenitor cell proliferation. J Biol Chem 2007;282: 25875-25883.

35 Seri B, Herrera DG, Gritti A, Ferron S, Collado L, Vescovi A, Garcia-Verdugo JM, Alvarez-Buylla A: Composition and organization of the SCZ: a large germinal layer containing neural stem cells in the adult mammalian brain. Cereb Cortex 2006;16 (suppl 1):I103I111.
36 Laskowski A, Howell OW, Sosunov AA, McKhann G, Gray WP: NPY mediates basal and seizure-induced proliferation in the subcallosal zone. Neuroreport 2007;18:10051008 .

-37 Nakagomi T, Taguchi A, Fujimori Y, Saino O, Nakano-Doi A, Kubo S, Gotoh A, Soma T, Yoshikawa H, Nishizaki T, Nakagomi N, Stern DM, Matsuyama T: Isolation and characterization of neural stem/progenitor cells from post-stroke cerebral cortex in mice. Eur J Neurosci 2009;29:1842-1852.

38 Burns KA, Murphy B, Danzer SC, Kuan CY: Developmental and post-injury cortical gliogenesis: a genetic fate-mapping study with nestin-creER mice. Glia 2009;57:1115-1129.

>39 Biran V, Joly LM, Heron A, Vernet A, Vega C, Mariani J, Renolleau S, Charriaut-Marlangue C: Glial activation in white matter following ischemia in the neonatal P7 rat brain. Exp Neurol 2006;199:103-112.

40 Segovia KN, McClure M, Moravec M, Luo NL, Wan Y, Gong X, Riddle A, Craig A, Struve J, Sherman LS, Back SA: Arrested oligodendrocyte lineage maturation in chronic perinatal white matter injury. Ann Neurol 2008;63:520-530

41 Sellers DL, Maris DO, Horner PJ: Postinjury niches induce temporal shifts in progenitor fates to direct lesion repair after spinal cord injury. J Neurosci 2009;29:6722-6733.

42 Guo F, Ma J, McCauley E, Bannerman P, Pleasure D: Early postnatal proteolipid promoter-expressing progenitors produce multilineage cells in vivo. J Neurosci 2009;29: 7256-7270.

43 Rothstein RP, Levison SW: Gray matter oligodendrocyte progenitors and neurons die caspase-3-mediated deaths subsequent to mild perinatal hypoxic/ischemic insults. Dev Neurosci 2005;27:149-159. 